\title{
Polimorfismo de los alelos de los antígenos de leucocitos humanos HLA-DRB1 y su asociación con la artritis reumatoidea juvenil en una muestra de niños mestizos colombianos
}

\author{
Gloria Garavito ${ }^{5}$, Clara Malagón ${ }^{2}$, Luis A. Ramírez ${ }^{3}$, Oscar F. De La Cruz ${ }^{3}$, Oscar Uribe ${ }^{3}$, \\ Edgar Navarro ${ }^{1}$, Antonio Iglesias ${ }^{4}$, Paz Martínez ${ }^{5}$, Dolores Jaraquemada ${ }^{5}$, Eduardo Egea ${ }^{1}$ \\ ${ }^{1}$ Laboratorio de Inmunología y Biología Molecular, Universidad del Norte, Barranquilla, Colombia. \\ ${ }^{2}$ Servicio de Reumatología, Clínica del Niño, ISS, Bogotá, D.C., Colombia. \\ ${ }^{3}$ Departamento de Reumatología, Universidad de Antioquia, Medellín, Colombia. \\ ${ }^{4}$ Departamento de Reumatología, Universidad Nacional de Colombia, Bogotá, D.C., Colombia. \\ ${ }^{5}$ Universidad Autónoma de Barcelona, Barcelona, España.
}

Se buscó tipificar molecularmente los alelos de los antígenos de leucocitos humanos HLADRB1 en un grupo de niños mestizos colombianos (población resultante de la mezcla genética de amrindios, europeos y africanos) con artritis reumatoidea juvenil (ARJ), así como analizar su frecuencia de expresión y compararla con sujetos clínicamente normales e investigar la asociación entre la frecuencia de los alelos con los diferentes subgrupos clínicos de ARJ. EI estudio involucró 65 pacientes con ARJ y 65 controles sanos. La tipificación de los alelos HLADRB1 se realizó por medio de la metodología de la reacción en cadena de la polimerasa con sondas de oligonucleótidos de secuencias específicas (PCR-SSOP), utilizando el protocolo recomendado por el XII International Histocompatibilty Workshop, realizado en St. Malo, París, en 1996. Los alelos HLADRB1* 1104 (prueba exacta de Fisher, PEF) $=0,013, \mathrm{OR}=16,79$, frecuencia etiológica $(\mathrm{FE})=0,93)$ y $\mathrm{DRB} 1 * 1602(\mathrm{PEF}=0,016, \mathrm{OR}=8,98, \mathrm{FE}=0,88)$ se evidenciaron como marcadores de susceptibilidad. HLA-DRB1*1501 ( $F P=0,466 ; p=0,005$ ) y HLA DRB1*1402 ( $F P=0,49 ; p=0,009)$ se comportaron como alelos asociados con protección. Al comparar las asociaciones entre alelos y los diferentes subgrupos clínicos se encontró asociación entre ARJ oligoarticular con HLA-DRB1* $1104(p=0,0034, \mathrm{OR}=41,53, \mathrm{FE}=0,97)$, la ARJ poliarticular se asoció con el alelo HLADRB1* $0404(p=0,012, \mathrm{OR}=8,75, \mathrm{FE}=0,88)$ y en el grupo sistémico, el alelo más expresado fue el HLA-DRB1*1602 $(p=0,005, \mathrm{OR}=21,33, \mathrm{FE}=0,95)$. La presencia de factor reumatoide estuvo asociado con los alelos HLA-DRB1*0407 $(p=0,05, \mathrm{OR}=11,2$, $\mathrm{FE}=0,45)$ y HLA-DRB1 ${ }^{*} 1302(p=0,02, \mathrm{OR}=22,8, \mathrm{FE}=0,63)$. En el grupo de pacientes con ANA+, sólo hubo significancia estadística para el alelo HLA-DRB1* 0701 ( $p=0,001, \mathrm{OR}=58, \mathrm{FE}=0,73$ ). Nuestros resultados sugieren que los genes del MHC en este subgrupo étnico inciden no sólo en la susceptibilidad a desarrollar ARJ sino también en la expresión clínica de la enfermedad.

Palabras clave: artritis reumatoide juvenil, alelos HLA-DR, polimorfismo, subtipos HLA-DRB1.

Polymorphism of human leukocyte antigen (HLA-DRB1) allelles and their association with juvenile rheumatoid arthritis in a group of Colombian mestizo children

Oligotypes of the human leukocyte antigen HLA Class II, DRB1 alleles were characterized at the molecular level in a group of Colombian children suffering juvenile rheumatoid arthritis (JRA). The distribution of these alleles was examined in a group of Colombian mestizo children (genetic admixture of Amerindians, Europeans and Africans) suffering from clinically distinct JRA subsets in order to detect HLA allele frequency differences in patients with different JRA subsets. A group of 65 patients with JRA and 65 controls were characterized for the subtypes of the HLA-DRB1 alleles using polymerase chain reaction with sequence-specific oligonucleotide probes (PCR-SSOP). The oligotyping protocol recommended by the 12th International Histocompatibility Workshop held in St. Malo, Paris, in 1996, was used. Subtype HLA-DRB1*1104 was the allele most strongly associated with susceptibility to JRA (Fisher's 
$p=0.013$, odds ratio $(O R)=16.79$, etiologic fraction $(E F)=0.93)$. HLA-DRB1*1602 was also associated with susceptibility to a lesser degree (Fisher's $p=0.016, O R=8.98, E F=0.88$ ). HLADRB1 alleles participating in JRA protection were HLA-DRB1*1501 (preventive fraction $(P F)=0.466, p=0.005)$ and HLA DRB1*1402 (PF=0.49, $p=0.009)$. The relationship between some HLA-DRB1 alleles and clinical features was also compared. The presence of rheumatic factor was associated with the alleles HLA-DRB1*0407 ( $p=0.05, \mathrm{OR}=11.2, \mathrm{EF}=0.45)$ and HLA$\mathrm{DRB} 1 * 1302(p=0.02, \mathrm{OR}=22.8, \mathrm{EF}=0.63)$. There was also an association between HLA$\mathrm{DRB} 1{ }^{*} 0701(p=0.001, \mathrm{OR}=58, \mathrm{EF}=0.73)$ with expressing $\mathrm{ANA}+$. We found that in the oligoarticular subset, the allele HLA-DRB1*1104 ( $p=0.0034, \mathrm{OR}=41.53, \mathrm{EF}=0.97)$ was the one expressed most commonly. In the poliarticular group, the alleles most frequently expressed were HLA-DRB $1{ }^{*} 0404$ (Fisher's $p=0.012, \mathrm{OR}=8.75, \mathrm{EF}=0.88$ ). In patients with systemic JRA, the HLA-DRB $1{ }^{*} 1602$ allele $(p=0.005, \mathrm{OR}=21.33, E F=0.95)$ was most frequent. These results suggested that the MHC genes of mestizo children influence not only the clinical expression of the disease, but also the susceptibility to its development.

Key words: juvenile rheumatoid arthritis, HLA-DR genes, MHC polymorphism, HLA DRB1 subtypes.

La artritis reumatoide juvenil (ARJ) constituye la causa más común de artritis crónica en niños y es la menos estudiada desde el punto de vista inmunogenético (1). Esta enfermedad corresponde a un grupo de cuadros clínicos heterogéneos con manifestaciones clínicas que varían en morbilidad, presentación y curso clínico $(2,3)$. A diferencia de la artritis reumatoide del adulto (AR), la ARJ se presenta clínicamente con tres subgrupos clínicos, lo cual la hace más interesante como modelo para estudiar desde el punto de vista inmunogenético, al permitir asociar marcadores del complejo mayor de histocompatibilidad (MHC) con la enfermedad.

Por definición, el inicio de la enfermedad ocurre antes de los 16 años de edad y está clasificada en tres grupos clínicos: ARJ oligoarticular, ARJ poliarticular y ARJ sistémica (4-8). El grupo de ARJ oligoarticular es el más frecuente $(50 \%$ de los casos) y en éste es frecuente el compromiso ocular, cuya manifestación clínica es la uveítis, la cual generalmente es subclínica $(6,9)$.

La literatura y los grupos que la estudian en el mundo están de acuerdo en que, desde el punto de vista inmunogenético, AR y ARJ son enfermedades diferentes (10-12).

\footnotetext{
Correspondencia:

Gloria Garavito, División Ciencias de la Salud, Laboratorio de Inmunología y Biología Molecular, Universidad del Norte, Km 5 Vía Sur América, Barranquilla, Colombia Tel.: 3509486; Fax: 3598852 ggaravito@uninorte.edu.co
}

Recibido: 20/11/02; aceptado: 23/05/03
Numerosos estudios se han llevado a cabo con diferentes poblaciones, tratando de asociar los diferentes subgrupos clínicos de ARJ con el polimorfismo del MHC. Se han encontrado variaciones según el grupo étnico estudiado y se han identificado varias asociaciones HLA. También se ha establecido que determinados alelos pueden jugar un papel importante como factor de riesgo para el desarrollo de ciertas complicaciones de la enfermedad o estar asociado con un gen protector. Estos hallazgos se han obtenido utilizando bien sea técnicas serológicas o tipificación del ADN (15-18). La mayoría de estos estudios se han llevado a cabo en europeos y norteamericanos, en especial, caucásicos y anglosajones. Estas investigaciones han mostrado datos polémicos debido al número restringido de pacientes estudiados $(13,14)$. El subgrupo clínico más estudiado es la ARJ oligoarticular. En éste se han encontrado asociaciones con HLA-DRB $1{ }^{*} 08$, DRB1 ${ }^{*} 11$ y DRB ${ }^{*} 13,(2,15-18)$. El alelo DRB $1{ }^{*} 07$ se ha encontrado disminuido en este grupo, lo que sugiere un papel protector $(17,19,20)$. En los pacientes con las características clínicas de ARJ poliarticular que son FR (+), la asociación más significativa es con el alelo HLA- DRB $1{ }^{*} 0404$. Este mismo alelo está implicado en AR, lo cual sugiriere que este subgrupo clínico pudiera ser el mismo de la $A R$ en el adulto de instalación temprana $(2,21,22)$. En el subgrupo poliarticular con FR negativo, se han asociado los alelos DRB1*01 y DRB1*08 $(17,22,23)$. El desarrollo de uveítis, la cual es más frecuente en pacientes con FR 
negativo y anticuerpos antinucleares positivo $\mathrm{ANA}+$, se ha informado asociación con los marcadores DRB1*11 y DRB1*13 $(2,15,22)$.

En este estudio, llevado a cabo en pacientes mestizos colombianos, encontramos el alelo HLADRB1*1104 asociado con susceptibilidad con ARJ $y$, en especial, al subgrupo clínico ARJ oligoarticular, asociación previamente descrita en caucásicos por otros autores. También encontramos una asociación entre la susceptibilidad de desarrollar la enfermedad con el alelo DRB1*1602, la cual es más frecuente en el fenotipo sistémico. Los alelos HLA-DRB1*1501 y DRB1*1402 se asociaron con protección.

El desarrollo de este trabajo permitió estudiar las asociaciones entre marcadores biológicos con las características clínicas de la población colombiana más representativa (mestizos) y sugerir marcadores de protección o susceptibilidad.

\section{Pacientes y métodos}

La muestra de pacientes estuvo conformada por 65 niños con diagnóstico de ARJ, todos mestizos colombianos en edades comprendidas entre 2 y 16 años de edad. Treinta y tres de ellos provenientes de la consulta reumatológica institucional del Hospital San Vicente de Paúl de Medellín y los 32 restantes de la Clínica del Niño del Instituto de los Seguros Sociales y de la Clínica Colsubsidio de Bogotá. Los pacientes se diagnosticaron de acuerdo con los criterios y la clasificación del American College of Rheumatology $(4,7)$. En todos ellos el inicio del cuadro clínico se dio antes de los 16 años. A todos se les practicaron marcadores serológicos de autoinmunidad y se les investigó la presencia de anticuerpos antinucleares usando la técnica de inmunofluorescencia con células HEP-2 y factor reumatoide por prueba de látex. Se sometieron a una valoración oftalmológica inicial y a controles periódicos (cada 4-6 meses) para detectar uveítis. Se conformaron subgrupos de 33 y 32 pacientes de cada ciudad, respectivamente. Previamente, los niños habían sido evaluados y vigilados clínicamente por un período no inferior a 6 meses antes de su ingreso al protocolo de la investigación. Toda la recolección de la información fue primaria y fue realizada por los investigadores clínicos y se registró en un formato de protocolo previamente elaborado para ello.

\section{Controles}

En el grupo control se seleccionaron 65 niños clínicamente normales, del mismo grupo étnico de los pacientes con ARJ. Estos niños no estaban relacionados familiarmente y presentaban igual distribución por sexo y edad. El área de procedencia era similar a la de los pacientes con ARJ. Los padres y abuelos de todos ellos eran originarios de esas áreas o de la ciudad asignada. Ninguno tenía un familiar cercano que trabajara en consultorios clínicos o áreas de prestación de servicios de salud. Ninguno tenía antecedentes personales ni familiares de alergia, enfermedades autoinmunes, psoriasis o espóndiloartropatías.

\section{Obtención del ADN}

A cada paciente y control se les tomaron las muestras biológicas a partir de sangre venosa periférica para extraer el ADN por la metodología de "extracción de sales" (salting out) (24).

\section{Tipificación molecular de los alelos HLA-DRB1}

Todas las muestras de los pacientes y controles fueron tipificadas para identificar los alelos HLADRB1 utilizando la técnica de reacción en cadena de la polimerasa (PCR) con oligonucleótidos de secuencia específica (PCR-SSOP). EI procedimiento usado fue el recomendado por el 12th International Histocompatibility Workshop Congress $(25,26)$. Los iniciadores y las sondas utilizadas en dicho protocolo permitieron una tipificación de alta resolución.

\section{Análisis estadístico}

Para el cálculo y la interpretación de la significancia estadística de las diferentes frecuencias alélicas y para el análisis de las asociaciones observadas, se utilizó la prueba de ji al cuadrado $\left(\chi^{2}\right)$, basada en una tabla de $2 \times 2$ con corrección de Mantel-Haenszel. En aquellos casos en el que la tabla de 2 x 2 contenía un valor menor de 5 , se utilizó la prueba exacta de Fisher (PEF), con un intervalo de confianza del $95 \%$. El OR se calculó para aquellos alelos que mostraron significancia estadística cuando se 
compararon con los del grupo control. La fracción etiológica y la fracción preventiva se calcularon para aquellos antígenos que mostraron asociación o estaban ausentes en la muestra de pacientes estudiada (27).

\section{Resultados}

\section{Características clínicas}

Las características clínicas de los pacientes incluidos en este estudio resultaron ser similares a aquéllas descritas por otras series publicadas en la literatura $(2,22,23,28,29,30-32)$. El grupo de edad en el cual se encontró el mayor número de pacientes (38 pacientes) fue el de escolares entre los 5 y 10 años de edad (60,3\%), seguido del grupo de 2 a 5 años $(22,2 \%)$. Los 11 pacientes restantes $(17,5 \%)$ tenían entre 11 y 16 años de edad. La literatura no aporta conclusiones respecto de la asociación entre alelos HLA y la instalación temprana de ARJ. Sólo existen datos obtenidos de análisis serológicos que asocian el HLA-DR4 con poliartritis de instalación temprana $(33,34)$. Aunque la entidad fue más prevalente entre mujeres $(60 \%)$, este hallazgo no fue estadísticamente significativo. La distribución del número de pacientes con relación al subgrupo clínico en el momento de la instalación del cuadro clínico mostró resultados iguales a los descritos por otros autores. De 65 pacientes, 26 se clasificaron como ARJ oligoarticular, 23 como ARJ poliarticular y los otros 16 tuvieron un cuadro clínico de ARJ de tipo sistémico. Del total de pacientes, sólo $6(9,2 \%)$ tuvieron ANA positivos. El $10,8 \%$ de la muestra (7 pacientes) tenía presencia de FR positivo. Se encontró desnutrición proteico-calórica y retardo del crecimiento entre los subgrupos clínicos poliarticular y sistémico. La desnutrición grado II fue la más frecuente y la ARJ poliarticular, el subgrupo clínico con mayor prevalencia $(34,7 \%)$ (figura 1).

No se encontró significación estadística cuando se buscó una asociación entre el curso clínico de esta enfermedad y la presencia de estos marcadores autoinmunes. Se destaca que de los 65 pacientes, sólo en 1 se diagnosticó uveítis. Éste pertenecía al subgrupo clínico poliarticular y su fenotipo fue tipificado como HLA-DRB1*1301/ ${ }^{*} 1601$.

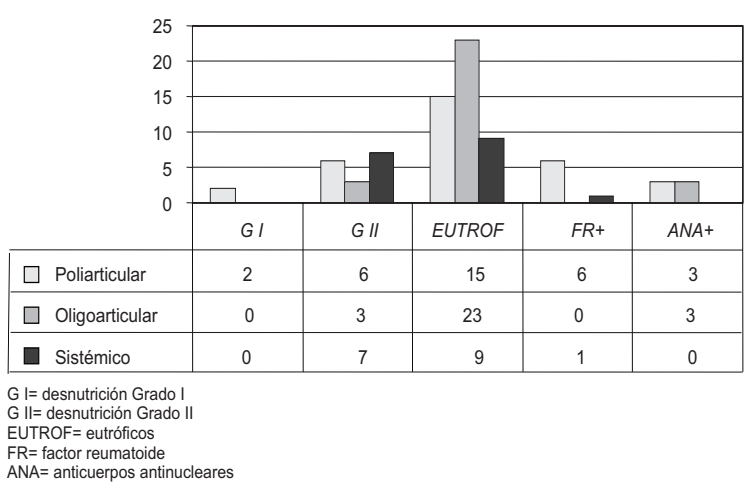

Figura 1. Características de la población estudiada.

\section{Tipificación molecular de los alelos HLA-DRB1}

Los resultados de la tipificación molecular para los alelos HLA-DRB1, tanto en los sujetos enfermos como en el grupo de control, se muestran en el cuadro 1 , en el que sólo se presentan los alelos más frecuentemente expresados, resaltando los que están significativamente incrementados o disminuidos. Los alelos DRB1 más frecuentemente expresados en los pacientes con ARJ fueron el DRB1*1104 (PEF=0,013; $\mathrm{OR}=16,79 ; \mathrm{IC} 95 \%=0,84-280,64 ; \mathrm{FE}=0,93)$ y el alelo DRB1 ${ }^{*} 1602$ (PEF=0,016; OR=8,98; IC95\% $=0,73-150,3 ; \mathrm{FE}=0,88)$, los cuales se asociaron con susceptibilidad.

Al comparar el alelo DR15 tanto en los controles sanos como en los pacientes, se observó una alta expresión en el grupo control $(p=0,0078$, $\mathrm{FP}=0,26$ ); el subtipo $\mathrm{DRB} 1{ }^{*} 1501$ fue el más significativo $(p=0,0048 ; \mathrm{IC} 95 \%=0,02-0,72$; $\mathrm{FP}=0,466)$. Igual patrón estadístico se observó en el alelo DR14 $(p=0,015 ; \mathrm{FP}=0,51)$, con el subtipo DRB1*1402 como el de mayor significancia ( $p=0,0087 ; \mathrm{IC} 95 \%=0-0,80 ; \mathrm{FP}=0,49)$. Los alelos DRB $1{ }^{*} 0405$ y DRB $1{ }^{*} 0407 \mathrm{se}$ expresaron de manera significativa en los pacientes con ARJ, pero al compararlos con los sujetos sanos no mostraron significancia estadística.

\section{Distribución de los alelos DRB1 en los diferentes subgrupos clínicos de ARJ}

En el subgrupo oligoarticular, el alelo que más se expresó fue el $\mathrm{DRB} 1{ }^{*} 1104 \quad(\mathrm{OR}=41,53$; 
Cuadro 1. Alelos HLA-DRB1 en mestizos colombianos con ARJ.

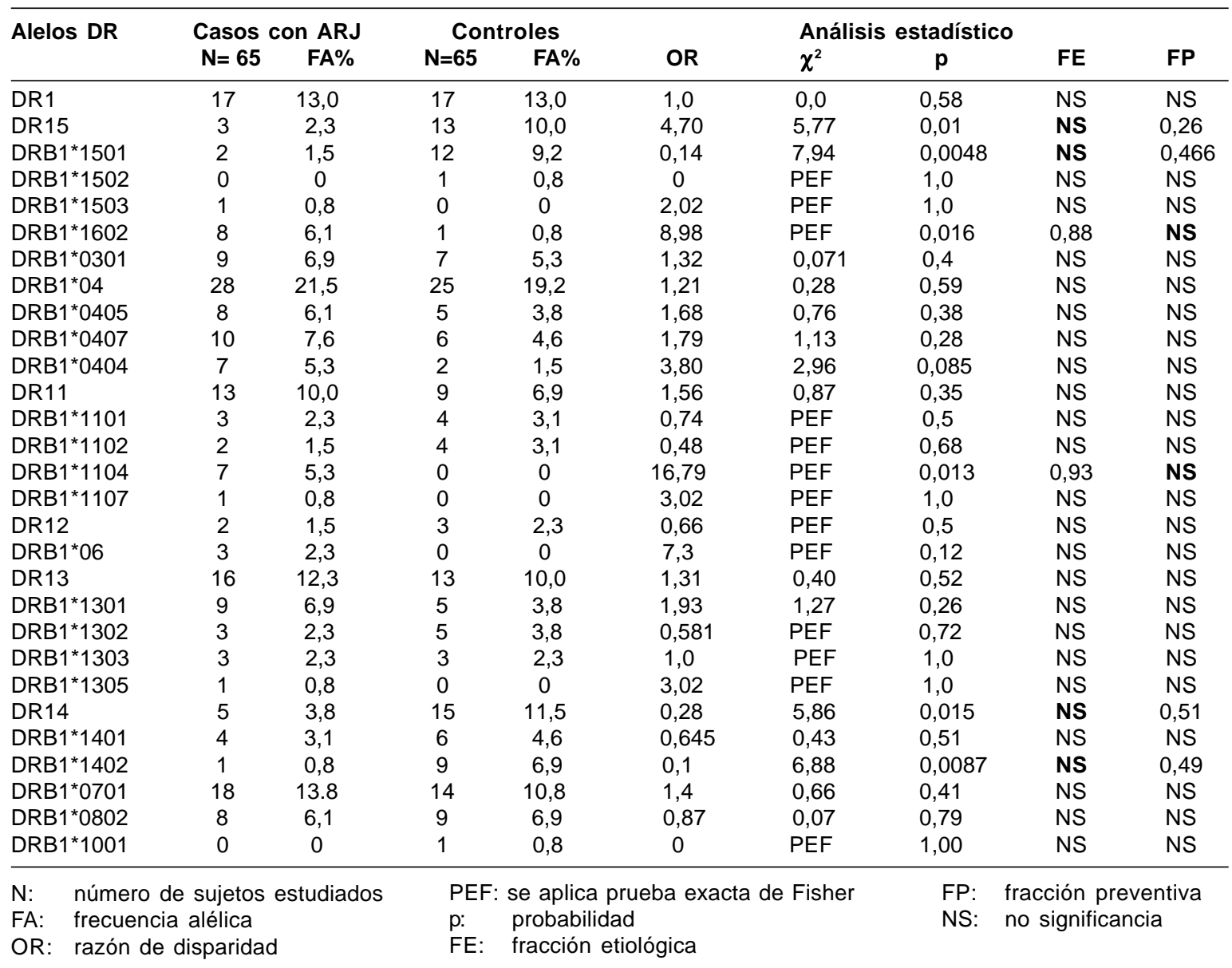

$p=0,00034)$. Este hallazgo es consistente con los informes previos de la literatura $(15,35,36)$. EI HLA$\mathrm{DRB} 1$ * 0404 (OR=8,750; $p=0,012)$ fue el que más se expresó en el subgrupo de pacientes con ARJ poliarticular. El subgrupo clínico de pacientes con manifestaciones de ARJ sistémica mostró una asociación con el incremento de la expresión del alelo DRB1* 1602 (OR=21,33; $\mathrm{p}=0,0048)$ (cuadro 2).

La presencia de ANA positivo se asoció con el alelo DRB1* 0701 ( $p=0,001 ; \mathrm{OR}=58 ; \mathrm{FE}=0,73)$. También se encontró un incremento significativo de los alelos DRB1 ${ }^{*} 0407(p=0,05 ; \mathrm{OR}=11,2 ; \mathrm{FE}$ $=0,45)$ y DRB $1 * 1302(p=0,02 ; \mathrm{OR}=22,8 ; \mathrm{FE}=0,63)$ en aquellos pacientes que tuvieron factor reumatoide positivo.

\section{Discusión}

En las enfermedades autoinmunes del humano se ha podido documentar una asociación entre el polimorfismo de los alelos HLA-clase I y clase II con susceptibilidad de desarrollar dichas enfermedades. De hecho, se ha postulado que en las enfermedades hereditarias complejas y poligénicas, la respuesta inmune dirigida contra antígenos propios es crucial en la etiopatogenia de la enfermedad y que esta respuesta estaría condicionada por una particular expresión o rearreglo de los genes en un individuo. De esta forma, un alelo HLA específico podría favorecer la presentación antigénica de un péptido artritogénico a un repertorio de TcR predeterminado genéticamente (37). 
Cuadro 2. Frecuencia alélica de los alelos HLA-DRB1 más frecuentemente expresados en diferentes subgrupos clínicos de mestizos colombianos con ARJ.

\begin{tabular}{|c|c|c|c|c|c|}
\hline \multirow{2}{*}{$\begin{array}{l}\text { Alelos } \\
\text { DRB1*1104 }\end{array}$} & \multirow{2}{*}{$\begin{array}{c}\begin{array}{c}\text { Controles } \\
\mathrm{N}=65\end{array} \\
\mathrm{~F}^{*}=0\end{array}$} & \multicolumn{4}{|c|}{$\begin{array}{l}\text { ARJ oligoarticular } \\
\qquad \mathrm{N}=26\end{array}$} \\
\hline & & $\begin{array}{c}F \\
\text { (6) } 23.07 \%\end{array}$ & $\begin{array}{c}\text { OR } \\
41.53\end{array}$ & $\begin{array}{c}p \\
0.00034\end{array}$ & $\begin{array}{l}\text { FE } \\
0.97\end{array}$ \\
\hline \multirow[t]{2}{*}{ DRB1*0404 } & $F^{*} \quad$ (2) $3.0 \%$ & \multicolumn{4}{|c|}{$\begin{array}{l}\text { ARJ poliarticular } \\
\qquad \mathrm{N}=23\end{array}$} \\
\hline & & $\begin{array}{c}F \\
(5) 21.73 \%\end{array}$ & $\begin{array}{c}\text { OR } \\
8.750\end{array}$ & $\begin{array}{c}p \\
0.012\end{array}$ & $\begin{array}{l}\mathrm{FE} \\
0.88\end{array}$ \\
\hline \multirow[t]{2}{*}{ DRB1*1602 } & $F^{*}(1) 1.5 \%$ & \multicolumn{4}{|c|}{$\begin{array}{l}\text { ARJ sistémica } \\
\qquad N=16\end{array}$} \\
\hline & & $\begin{array}{c}F \\
(4) 25 \%\end{array}$ & $\begin{array}{c}\text { OR } \\
21.33\end{array}$ & $\begin{array}{c}p \\
0.0048\end{array}$ & $\begin{array}{c}\mathrm{FE} \\
0.95\end{array}$ \\
\hline
\end{tabular}

$\mathrm{N}$ : número de sujetos

FE: fracción etiológica

$\mathrm{F}$ : frecuencia alélica

A diferencia de la artritis reumatoide del adulto, la artritis reumatoide juvenil es una enfermedad heterogénea desde el punto de vista clínico, con un curso variable, en la que se definen subgrupos clínicos claramente diferentes (38). El componente genético en la patogénesis de artritis reumatoide del adulto ha sido bastante investigado, a diferencia de la artritis reumatoide juvenil, en la cual los estudios al respecto no son concluyentes; sin embargo, es claro que los genes y los mecanismos que condicionan la predisposición o protección en ARJ son diferentes (39).

La ARJ es una enfermedad compleja, poligénica, en la cual se han podido documentar asociaciones no sólo con los genes asociados con el MHC sino también con muchos otros marcadores tales como los genes promotores de IL-1, IL-6 e IL-10, TNF alfa/beta y el polimorfismo de la región variable 6.1 de TcR, entre otros(40). Aunque las asociaciones más consistentes y reproducibles han sido las descritas con el polimorfismo de los genes $\mathrm{MHC}$, los otros marcadores genéticos complican el análisis de las bases genéticas de esta enfermedad. Además, es evidente que no existen razones para suponer que la ARJ sea diferente de las otras enfermedades autoinmunes en las cuales se han podido documentar varios sistemas genéticos esparcidos por diferentes cromosomas asociados con la susceptibilidad y expresión de la enfermedad (41).
A pesar de muchos estudios encaminados a asociar antígenos de leucocitos humanos y artritis reumatoide juvenil (42), no está claro aún cuáles de los genes del complejo mayor de histocompatibilidad están directamente involucrados en la patogénesis de esta enfermedad, la que, por ser una enfermedad heterogénea, hace más compleja su asociación con los alelos HLA. Todos los estudios publicados se han desarrollado en pacientes caucásicos y anglosajones mas no en grupos étnicos mestizos. Hasta la fecha no había información de estudios inmunogenéticos en pacientes latinoamericanos, ni mucho menos en colombianos.

El subgrupo oligoarticular ha sido el más estudiado desde el punto de vista genético $(43,28)$ y el alelo DRB1*1104 es el que más significado estadístico ha mostrado, en particular en el subgrupo ARJ oligoarticular de instalación temprana que desarrolla uveítis. Otro alelo de asociación es DRB $1{ }^{*} 001 / 3$ con ARJ poliarticular y FR negativo. En los pacientes con ARJ poliarticular y FR positivo, (manifestación clínica que más se asemeja a $A R$ ), la asociación más descrita es con HLA-DRB1*04 (10-12). Algunos análisis de secuencia de los alelos DR4 revelan una frecuencia alta con DRB $1{ }^{*} 0401$ y DRB $1{ }^{*} 0404$ en caucasoides y con DRB ${ }^{*} 0405$ en pacientes japoneses (43), concepto que refuerza la relación 
estrecha entre AR y ARJ poliarticular con FR positivo $(10,43)$.

En este estudio describimos en nuestra población mestiza el alelo HLA-DRB1*1104 (PEF=0,011; $\mathrm{OR}=14,34 ; \mathrm{FE}=0,93$ ) como un marcador que se muestra asociado con un gen de susceptibilidad para ARJ. Este hallazgo es significativo, ya que es el primer trabajo en población mestiza que reproduce este marcador genético como un gen asociado con susceptibilidad en la población en general $(9,15,36)$. Igualmente, este mismo alelo fue el único marcador de susceptibilidad que mostró franca significación estadística en los pacientes del fenotipo oligoarticular ( $\mathrm{OR}=41,63$; $p=0,00035 ; \mathrm{FE}=0,97)$. También encontramos una asociación de susceptibilidad entre el alelo DRB $1{ }^{*} 1602$ y el fenotipo sistémico $(p=0,0048$; OR $=21,33 ; \mathrm{FE}=0,95$ ). Este último hallazgo es diferente de los publicados por otros autores, quienes no encontraron asociación alguna de este alelo con este fenotipo (29). Este mismo alelo fue descrito asociado con la enfermedad de Takayasu en mestizos de nuestro país (44).

Los alelos HLA DRB1*0405 y DRB1*0407 se encontraron mayormente expresados en los pacientes (28 de 65); sin embargo, al compararlos con los sujetos controles no se encontró significación estadística. Probablemente, la explicación de este hallazgo sea el hecho de que estos alelos son frecuentes en nuestra población mestiza (45).

La frecuencia de expresión del subtipo HLADRB $1{ }^{*} 0404$ mostró una significancia estadística con el fenotipo ARJ poliarticular $(p=0,012$; $\mathrm{OR}=8,75 ; \mathrm{FE}=0,88$ ), lo que coincide con lo encontrado por otros autores al estudiar otros grupos étnicos $(2,10-12,17,31)$, comportándose también como un marcador de susceptibilidad.

Los alelos HLA-DRB1 ${ }^{*} 1501$ ( $p=0,0048 ; O R=0,14$; $\mathrm{FP}=0,46)$ y DRB1*1402 $(p=0,0087 ; \mathrm{OR}=0,1 ; \mathrm{FP}$ $=0,49$ ) se comportan como marcadores de protección.

Desde el punto de vista inmunogenético, nuestros resultados reproducen los encontrados previamente por nosotros y otros autores (46-48), en los cuales se muestra que nuestra carga genética es una mezcla de genes de amerindios, caucásicos y negros, lo que refleja la carga genética que se ha establecido en esta parte de Suramérica en los últimos 6 siglos. Al asociar nuestros marcadores, alelos del HLA-DRB1, con el subgrupo sistémico, los datos no son estrictamente comparables con los encontrados por diferentes grupos de investigadores debido, probablemente, a que los datos obtenidos por ellos provienen de diferentes grupos de población (32).

A pesar del número relativamente pequeño de pacientes, en cada subgrupo clínico estudiado en nuestra serie, todas las asociaciones encontradas muestran un evidente valor estadístico. Nuestros resultados concuerdan con los datos publicados no sólo en la literatura sino también en las conclusiones del componente de ARJ del XII Taller Internacional de Inmunogenética y Trasplante (49). A pesar de que en nuestro trabajo la relación entre casos y controles es de 1:1, lo que plantea la posibilidad de error tipo 1, los valores de las fracciones etiológicas y de prevención muestran datos de demostrada asociación estadística.

Si las asociaciones descritas en este estudio pudieran reproducirse en otros grupos étnicos mestizos, con similares características demográficas, podría plantearse la hipótesis de que los mecanismos de susceptibilidad ligados al polimorfismo de ciertos genes del complejo mayor de histocompatibilidad (MHC) y la función de sus productos proteicos, los antígenos HLA, en el complejo trimolecular TcR-péptido-MHC pudieran participar en la susceptibilidad y en la expresión clínica de esta enfermedad.

De hecho, los resultados de este trabajo permiten la descripción, por primera vez en la población colombiana más representativa (mestizos), de la asociación entre marcadores MHC con las características clínicas de los tres subgrupos en ARJ y sugiere marcadores de protección o susceptibilidad.

\section{Agradecimientos}

Expresamos nuestros agradecimientos a todos los pacientes que participaron en este estudio y a Ana Sofía Moreno Woo, por el soporte técnico que demandó el proyecto. Esta investigación fue financiada parcialmente con fondos de Colciencias, 
código 1215-04-28-96 y de la Universidad del Norte, Dirección de Investigaciones y Proyectos.

\section{Referencias}

1. Grom AA, Giannini EH, Glass DN. Current comment: juvenile rheumatoid arthritis and the trimolecular complex (HLA T cell receptor and antigen). Differences from rheumatoid arthritis. Arthritis Rheum 1994;37:601-7.

2. Nepom B. The immunogenetics of juvenile rheumatoid arthritis. Rheum Dis Clin North America 1991;17:82542.

3. Cassidy JT, Levinson JE, Brewer EJ. The development of classification criteria for children with juvenile rheumatoid arthritis. Bull Rheum Dis 1989; 38:11-7.

4. Brewer EJ, Bass JC, Cassidy JT, et al. Criteria for the classification of juvenile rheumatoid arthritis. Bull Rheum Dis 1972;23:712-9.

5. Cassidy JT. Rheumatic diseases of the childhood. En: Kelley WN, Harris ED, Ruddy S, Sledge CB, editors. Textbook of rheumatology. Philadelphia: Saunders Eds.; 1993. p.1189-208.

6. Pachman LM, Poznanski AK. Juvenile rheumatoid arthritis. En: McCarty D, Coopman W, editors. Arthritis and allied conditions. Philadelphia: Lea \& Febiger; 1993 p.1021-38.

7. Brewer EJ, Bass J, Baum J, Cassidy JT, Fink CW, Jacobs J, et al. Current proposed revision of JRA criteria. Arthritis Rheum 1977;20:195-9.

8. Sakkas LI, Platsoucas CD. Immunopathogenesis of juvenile rheumatoid arthritis: role of T cells and MHC. Immunological Research 1995;14:218-36.

9. Malagon C, Van Kerckhove C, Giannini EH, Taylor J, Lovell DJ, Levinson JE, et al. The iridocyclitis of early onset pauciarticular juvenile rheumatoid arthritis: Outcome in immunogenetically characterized patients. J Rheumatol 1992;19:160-3.

10. Stastny P. Association of the B cell alloantigen DRw4 with rheumatoid arthritis. N Engl J Med 1978;298:867-71.

11. Nepom GT, Byers P, Seyfried C, Healey LA, Wilske KR, Stage D, et al. HLA genes associated with rheumatoid arthritis. Arthritis Rheum 1989;32:15-21.

12. Wordsworth BP, Lanchbury JSS, Sakkas LI, Welsh KI, Panayi GS, Bell JL. HLA-DR4 subtype frequencies in rheumatoid arthritis indicate that DRB1 is the major susceptibility locus within the HLA Class II region. Proc Natl Acad Sci USA 1989;86:10049-53.

13. Miller ML, Aaron S, Jackson J, Fraser P, Cairns L, Hoch S, et al. HLA gene frequencies in children and adults with systemic onset juvenile rheumatoid arthritis. Arthritis Rheum 1985;28:146-50.

14. Desaymard C, Kaplan C, Fournier C, Manigne P, Hayem F, Kahn MF. Major histocompatibility complex markers and disease heterogeneity in one hundred eight patients with systemic onset juvenile chronic arthritis. Revue Du Rheumatism (English edition) 1996;63-9.

15. Melin-Aldana H, Giannini EH, Taylor J, Lovell DJ, Levinson JE, Passo MH, et al. Human leukocyte antigen DRB1*1104 in the chronic iridiocyclitis of early onset pauciarticular juvenile rheumatoid arthritis. $J$ Pediatric 1992;121:56-60.

16. Brunner HI, Ivaskova E, Haas JP, Andreas A, Keller E, Hoza J, et al. Class I associations and frequencies of class II HLA-DRB alleles by RFLP analysis in children with rheumatoid-factor-negative juvenile chronic arthritis. Rheumatol Int 1993;13:83-8.

17. Ploski R, Vinjie O, Ronningen KS, Spurkland A, Sorskaar D, Vartdal F, et al. HLA Class II alleles and heterogeneity of juvenile chronic arthritis: DRB $1{ }^{*} 0101$ may define subset of the disease. Arthritis Rheum 1993;36:465-72.

18. Scholz S, Albert ED. Immunogenetic aspects of juvenile chronic arthritis. Clin Exp Rheumatol 1993;11:S37-41.

19. Fernandez-Vina M, Fink CW, Stastny P. HLA antigens in juvenile arthritis. Pauciarticular and polyarticular juvenile arthritis are immunogenetically distinct. Arthritis Rheum 1990;33:1787-94.

20. Ploski R. Immunogenetic polymorphism and disease mechanism in juvenile chronic arthritis. Revue Du Rheumatism (English edition) 1997;4127S-30S.

21. Forre O, Dobloug J, Hoyeral H, Kass E, Thorsby E. $\mathrm{HLA}$-antigens in rheumatoid arthritis and juvenile rheumatoid arthritis. Arthritis Rheum 1983;26:35-8.

22. Fink CW, Fernandez-Vina M, Statsny P. Clinical and genetic evidence that juvenile chronic arthritis is not a single disease. Pediatr Clin North Am 1995;42:1155-69.

23. Fernandez-Viña M, Fink CW, Stastny P. HLA associations in juvenile arthritis. Clin Exp Rheumatol 1994; 12:205-14.

24. Miller SA, Dykes DD, Plosky HF. A simple salting out procedure for extracting DNA from human nucleated cells. Nucleic Acids Res 1988;16:1215.

25. Olerup $\mathbf{O}$, Zetterquist H. HLA-DR typing by PCR amplification with sequence-specific primers (PCR-SSP) in 2 hours: an alternative to serological DR typing in clinical practice including donor-recipient matching in cadaveric transplantation. Tissue Antigens 1992;39:225-35.

26. Tiercy JM, Grundschober C, Jeannet M, Mach BA. Comprehensive HLA-DRB, DQB and DPB oligotyping procedure by hybridization with sequence-specific oligonucleotide probes. En: KM Hui, Bidwell JL, editors. Handbook of HLA- typing technique. Florida: CRC Press; 1993. p.117.

27. Woolf B. On estimating the relation between blood group and disease. Ann Hum Genet 1956;20:309-11.

28. Donn R, Pepper L, Carthy D. HLA-DPB1*0201 and DRB1*1104 are reduced in Greek pauciarticular-juvenile 
chronic arthritis patients. Clin Exp Rheumatol 1993; 11:S74.

29. Pratsidou-Gertsi P, Kanakoudi-Tsakalidou F, Spyropoulou M, Germenis A, Adam K, Taparkou A, et al. Nationwide collaborative study of HLA class II associations with distinct types of juvenile chronic arthritis (JCA) in Greece. Eur J Immunogenet 1999;26:299-310.

30. Alsaeid K, Haider MZ, Kamal H, Srivastva BS, Ayoub EM. Prevalence of human antigen (HLA) DRB1 alleles in Kuwaiti children with juvenile rheumatoid arthritis. Eur J Immunogenet 2002;29:1-5.

31. Morling N, Friis J, Fugger L,Georgsen J, Heilmann C, Pedersen FK, et al. DNA polymorphism of HLA class II genes in pauciarticular juvenile rheumatoid arthritis. Tissue Antigens 1991;38:16-23.

32. Prieur AM, Stavropoulos-Giokas C, Germenis A, Syropoulou M, Pratsidou P, Kanakoudi F, et al. Juvenile chronic arthritis (JCA): 12th International Histocompatibility Workshop study. En: Charron DJ, editor. HLA in medicine: genetic diversity of HLA. France: EDK Serves;1997. p. 398-407.

33. Murray KJ, Moroldo MB, Donnelly P, Prahalad S, Passo MH, Giannini EH, et al. Age-specific effects of juvenile rheumatoid arthritis-associated HLA alleles. Arthritis Rheum 1999;42:1843-53.

34. Jaraquemada D, Ollier W, Awad J, Young A, Silman A, Roitt M, et al. HLA and rheumatoid arthritis: a combined analysis of 440 British patients. Ann Rheum Dis 1986;45:627-36.

35. Ollier W,Thomson W. Population genetics of rheumatoid arthritis. Rheum Dis Clin North Am 1992;18:741-59.

36. Haas JP, Thuckenbrodt H, Paul C, Hoza J, Scholz S, Albert ED. Subtypes of HLA DRB $1{ }^{*} 03,{ }^{*} 08,{ }^{*} 11,{ }^{*} 12,{ }^{*} 13$ and *14 in early onset pauciarticular juvenile chronic arthritis (EOPA) with and without iridocyclitis. Clin Expert Rheumatol 1994;12:S7-S14.

37. Heard R. HLA and autoimmune disease. En: Lechler R, editor. HLA and disease. London: Academic Press; 1994. p.123-52.

38. Prieur AM, Petty RE. Definitions and classifications of chronic arthritis in children. En: Arthritis in children and adolescent. Clinical Pediatrics. London: Bailleres Tindall; 1993. p.124-30.

39. Stastny P, Fink CW. Different HLA-D associations in adult and juvenile rheumatoid arthritis. J Clin Invest 1979; 63:124-30.

40. Glass DN, Giannini EH. Juvenile rheumatoid arthritis as a complex genetic trait. Arthritis Rheum 1999; 42:2261-8.
41. Davies JL, Kawaguchi Y, Bennet SI, Copeman JB, Cordell HJ, Pritchard LE, et al. A genome-wide search for human type I diabetes susceptibility genes. Nature 1994;371:130-6.

42. Stastny $\mathbf{P}$, Xiaojang G, Fernandez $\mathbf{M}$. Immunogenetics of rheumatoid arthritis and juvenile arthritis. Medicine 1991;82:7-8.

43. Okubo H, Itou K, Tanka S, Watanabe N, Kashiwagi N, Obata F. Analysis of the HLA-DR gene frequencies in Japanese cases of juvenile rheumatoid arthritis by oligonucleotoide DNA typing. Rheumatol Int 1993; 13:65-9.

44. Salazar M, Varela A, Ramírez LA, Uribe O, Vásquez E, Egea E, et al. Association for HLA-DRB1*1602 and DRB1*1001 with Takayasu arteritis in Colombian mestizos as markers of Amerindian ancestry. Int $\mathrm{J}$ Cardiol 2000;75:S113-6.

45. Petz-Erler ML, Gorodezky C, Layrisse Z, Klitz W, Egea E, Garavito G, et al. Anthropology report for Region Latin-America: Amerindian and admixed populations. Anthropology report for Region Latin-America. En: Charron D, editor. Genetic diversity of HLA: functional and medical implication. Paris: EDK, Medical and Scientific International publishers; 1997. p.337-45.

46. Trachtenberg EA, Erlich HA, Hollenbach J, Keyeux G, Bernal J, Klitz W. HLA class II variation and linkage disequilibrium in nine Amerindian and three American populations from Colombia. Results of Expedición Humana. En: Charron D, editor. HLA genetic diversity of HLA functional and medical implication. Paris: EDK; 1997. p.200-2.

47. Silvera-Redondo C, Gomez-Casado E, MartinezLaso J, Egea E, Garavito G, Varela P, et al. A new HLA$\mathrm{Cw}$ allele $\left(\mathrm{CW}^{*} 0808\right)$ found in a Colombian mestizo individual possibly generated by an intralocus/interloci gene conversion. Immunogenetics 2000;51:1053-7.

48. Yunis JJ, Yunis EJ, Yunis E. Genetic relationship of the Guambino, Paez, and Ingano Amerindians of southwest Colombia using major histocompatibility complex class. II. Haplotypes and blood groups. Hum Immunol 2001;62:970-8.

49. Prieur AM, Stavropoulos-Giokas C, Germenis A, Spyropoulou M, Pratsidou $P$, Kanakoudi $F$, et al. Juvenile chronic arthritis (JCA): 12th International Histocompatibility Workshop Study. En: Charron DJ, editor. HLA in medicine: genetic diversity of HLA. France: EDK Serves; 1997. p.398-407. 